06.3

\title{
Радиопоглощающий метаматериал и экран антенны на его основе
}

\author{
(ㄱ Р.О. Рязанцев, Ю.П. Саломатов, С.В. Поленга \\ Сибирский фредеральный университет, Красноярск, Россия \\ E-mail: ryazantsevro@mail.ru
}

Поступило в Редакцию 2 июля 2021 г.

В окончательной редакции 20 августа 2021 г.

Принято к публикации 20 августа 2021 г.

\begin{abstract}
Исследован радиопоглощающий метаматериал, располагаемый вблизи сплошной металлической поверхности, и разработана конструкция антенны для приема спутниковых навигационных сигналов с использованием предлагаемого материала в составе поглощающего экрана. Разработана структура, исследованы частотные характеристики методом конечных элементов в частотной области, установлены рабочие размеры элементов исследуемого метаматериала для применения в составе поглощающего экрана. Выполнены моделирование и анализ диаграммы направленности для антенны с поглощающим экраном в сравнении с антенной на классическом высокоимпедансном экране. Сделаны выводы о применимости предлагаемой структуры метаматериала.
\end{abstract}

Ключевые слова: антенна ГНСС, радиопоглощающий метаматериал, высокоимпедансный экран.

DOI: 10.21883/PJTF.2021.23.51778.18947

Метаматериалы находят все большее применение в антенной технике. В частности, с использованием искусственных материалов выполняются экраны высокоточных антенн для приема сигналов глобальных навигационных спутниковых систем (ГНСС) [1]. Техническая реализация метаматериала основывается на классических работах $[2,3]$, и в таком случае метаматериал представляет собой комбинацию разомкнутых совмещенных кольцевых резонаторов и близкорасположенных линейных проводников. Данная среда позволяет создать одновременно отрицательные магнитную и диэлектрическую эффективные проницаемости и, что является интересным в свете данного исследования, работать с электромагнитной волной без значительных отражений от границы раздела среды. Использование такой реализации в виде решетки единичных элементов в пространстве, но с интегрированными поглощающими элементами возможно и известно [4]. Однако непосредственное использование такого варианта для работы в составе антенны ГНСС оказалось невозможным вследствие необходимости работы метаматериала в непосредственной близости от металлического экрана при нормальном к экрану типе поляризации, которая подлежит поглощению при небольших размерах единичного элемента метаматериала.

В настоящей работе исследован поглощающий метаматериал с целью улучшения электрических характеристик антенн при применении его в составе экранов антенных конструкций.

Для формирования специальных свойств экранов антенн ГНСС также известно применение структур Electromagnetic Bandgap (EBG), в том числе с использованием свойств метаматериалов [5], однако это сопряжено с технологическими трудностями, заключающимися в необходимости прямого электрического контакта структуры и металлического экрана.
Поглощающий метаматериал, разработанный по результатам исследования, выполнен по типу метаматериалов на основе разомкнутых кольцевых резонаторов, однако имеет особенности в том смысле, что не требует непосредственного контакта проводящих элементов с металлическим экраном и не содержит протяженных линейных проводников в топологии структуры.

В результате исследований был обнаружен эффект, заключающийся в том, что при соответствующей ориентации однокольцевого резонатора вблизи экрана существует область частот, в которой рассматриваемая периодическая структура обладает свойствами метаматериала. При этом разрез проводящего кольца ориентирован к экрану, а электромагнитное взаимодействие структуры кольца осуществляется с полем падающей волны, поляризация которой ортогональна экрану. Нагрузка резонаторного кольца резистором позволяет осуществлять поглощение электромагнитной волны, при этом свойства метаматериала позволяют осуществлять поглощение в отсутствие значительного уровня отражения сигнала. Единичная ячейка структуры представлена на рис. $1, a$. Кольцевые резонаторы могут быть изготовлены с помощью печатной технологии на распространенном диэлектрическом материале FR-4. B качестве резисторов могут быть использованы промышленные тонкопленочные компоненты поверхностного монтажа, применимость которых оговаривается производителем для работы на частотах порядка единиц и десятков гигагерц [6].

Расчет параметров отражения и поглощения выполнен путем анализа периодической структуры посредством моделирования методом конечных элементов в фиксированных частотных точках. Центральной рабочей частотой поглощения в исследовании была выбрана $1575 \mathrm{MHz}$ - центральная частота одного из диапазонов ГНСС. Исходя из графиков $S$-параметров модели эле- 

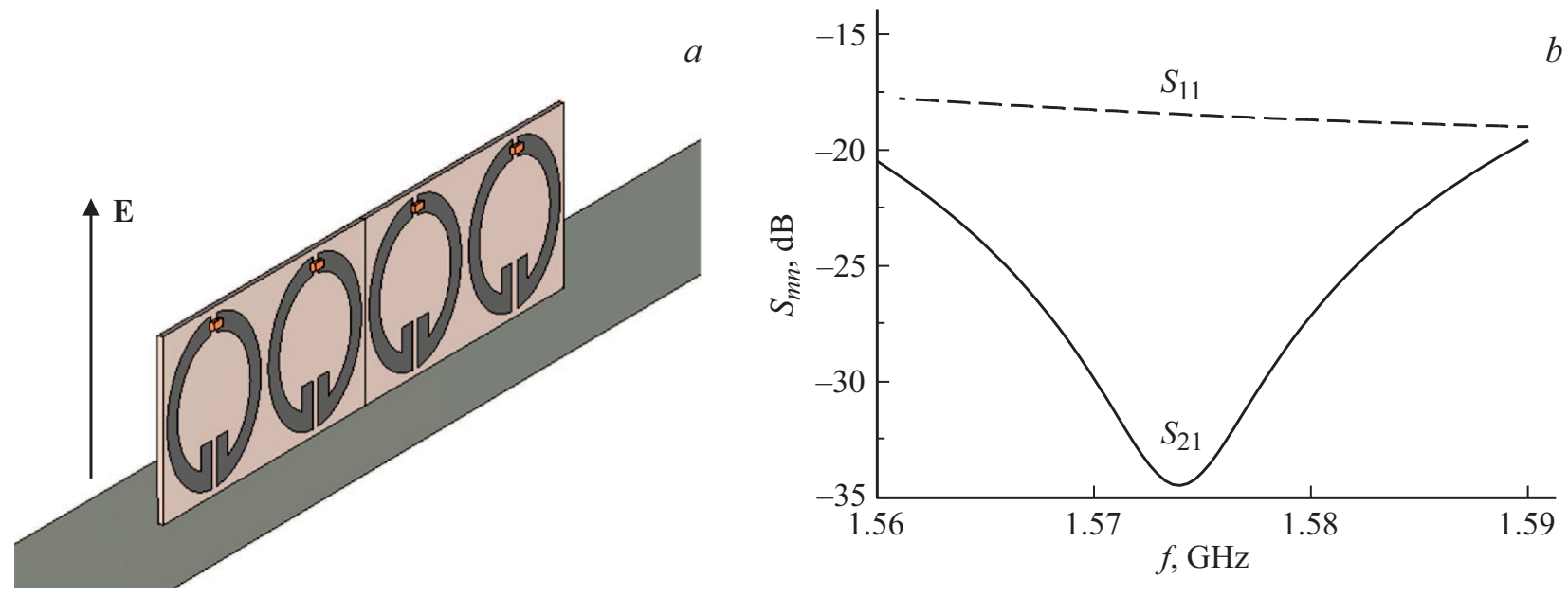

Рис. 1. $a-$ элементарная ячейка для расчета электромагнитных свойств структуры. $b-$ частотная зависимость $S$-параметров модели элементарной ячейки исследуемого метаматериала.

ментарной ячейки поглощающей структуры (рис. 1,b) можно утверждать, что исследуемая периодическая структура демонстрирует низкий уровень коэффициента отражения и низкий уровень коэффициента прохождения вблизи резонансной частоты, что эквивалентно высокому значению коэффициента поглощения.

В исследовании были рассчитаны параметры метаматериала, такие как относительные диэлектрическая и магнитная проницаемости. Для вычисления данных параметров использовались выражения из работы [7]. По итогам расчета эквиваленты действительных компонент на центральной рабочей частоте составили $\varepsilon=-1.6$, $\mu=-1.1$. Результаты расчета параметров относительных проницаемостей были верифицированы через анализ модели ячейки в тех же граничных условиях, состоящей из заполненного объема сплошного материала с заданными значениями проницаемостей.

В рассматриваемом случае с целью увеличения коэффициента эллиптичности диаграммы направленности антенны при работе на углах, близких к горизонту, предполагается поглощение поляризационной составляющей, перпендикулярной плоскости экрана антенны, подобно тому, как в классических антеннах ГНСС с гофрированным фланцем (choke ring) осуществляется подавление распространения $E$-компоненты падающей электромагнитной волны [8].

Ряды резонансных поглощающих элементов (32 пластины) метаматериала, расставленные по металлическому экрану квадрупольной антенны, составляют поглощающий экран, который предназначен для поглощения компоненты поля антенны с поляризацией, нормальной к экрану. Изометрический вид антенны для приема сигналов ГНСС с поглощающим экраном представлен на рис. 2, $a$.

Диаграммы направленности (ДН) основной и кросс-поляризационной составляющих для круговой поляризации антенны, экран которой выполнен в виде гофрированного металлического фланца, и антенны с исследуемым поглощающим метаматериалом в целом схожи (рис. 2,b). Значительная разница наблюдается за пределом рабочих углов, около направления в $180^{\circ}$, где антенна с поглощающим экраном имеет значительно больший $(\sim-10 \mathrm{dBi})$ уровень приема кросс-поляризованной составляющей. Однако данный факт ожидаемо не привнесет ухудшений в точность определения координат при приеме сигналов ГНСС при геодезических приложениях.

Более информативным является сравнение зависимостей коэффициента эллиптичности (axial ratio) для антенн с экранами различного типа, которые приведены на рис. 3 (в том числе для антенны с простым сплошным металлическим экраном - flat metal).

Известно, что при рассмотрении работы антенн высокоточного применения для приема сигналов ГНСС наиболее критичной проблемой являются многолучевое распространение [9] и подавление приема отраженных сигналов. В наиболее критичном диапазоне углов модель антенны с предлагаемым поглощающим экраном демонстрирует некоторое преимущество: коэффициент эллиптичности для углов ДН вблизи $90^{\circ}$ составляет около 0.78 , тогда как для антенны с металлическим гофрированным фланцем (три кольца) он равен 0.68 .

Антенна с предлагаемым типом поглощающего экрана отличается меньшими размерами: высота экрана предлагаемого типа составляет $19 \mathrm{~mm}$, в случае антенны с гофрированным фланцем - $48 \mathrm{~mm}$. Высота антенны с экраном предлагаемого типа определяется высотой излучателя и в модели составляет $40 \mathrm{~mm}$, высота антенны с фланцем определяется суммой высот излучателя и фланца и в модели составляет $88 \mathrm{~mm}$. Диаметры экранов антенн были выбраны близкими для обеспечения корректности сравнения (270 и $280 \mathrm{~mm}$ для антенны с метаматериалом и для антенны с фланцем соответственно).

Таким образом, исследования показывают, что реализация экрана антенны для приема сигналов ГНСС с использованием разработанного поглощающего мета- 

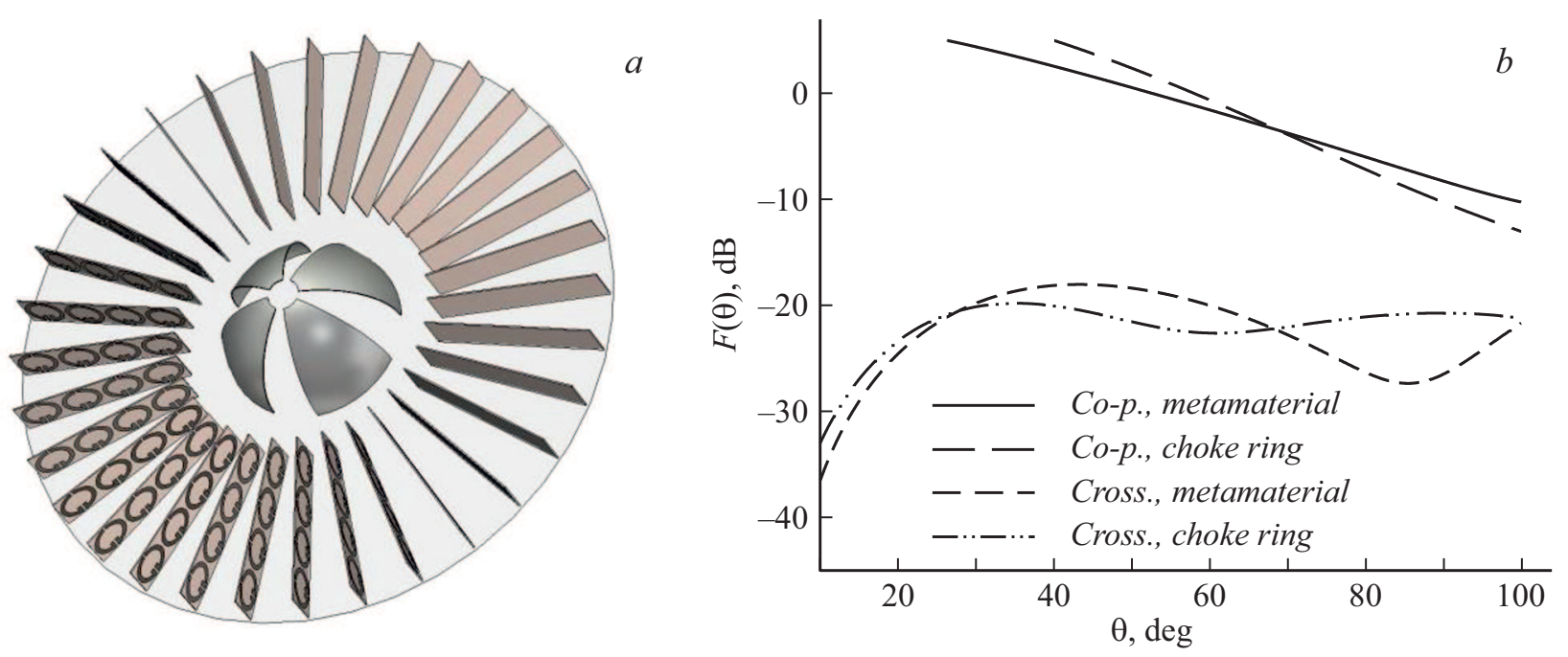

Рис. 2. $a-$ изометрический вид антенны для приема сигналов ГНСС с поглощающим экраном на основе исследуемого метаматериала. $b-$ ДН антенн в секторе рабочих углов. Co-p. - основная поляризация, Cross. - кросс-поляризация.

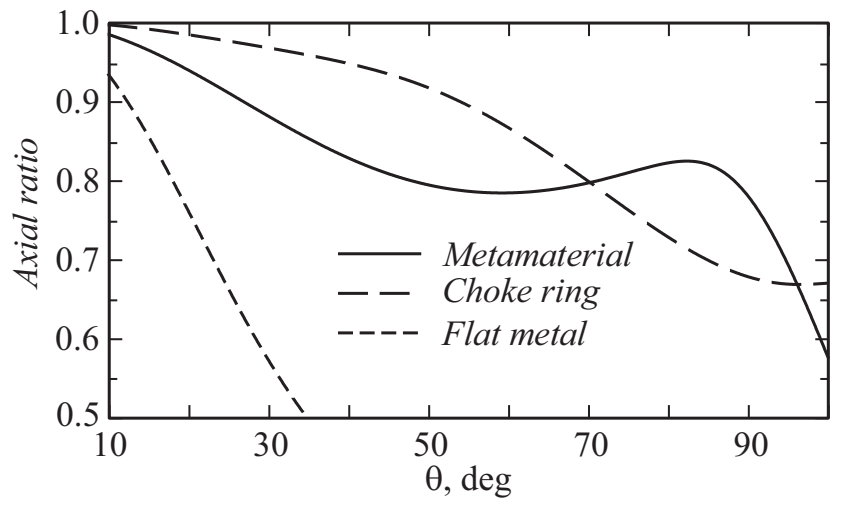

Рис. 3. Зависимости коэффициента эллиптичности для антенн с различными типами экранов.

материала является конкурентоспособной и позволяет при высоком уровне электрических характеристик, как минимум, улучшить массогабаритные параметры подобных антенн. Также такой вариант поглощающего экрана является экономически целесообразным вследствие использования распространенного и недорогого диэлектрического материала FR-4 и технологии изготовления печатных плат.

Представленное исследование имеет перспективы в части расширения рабочей полосы частот метаматериала (либо формирования отдельной второй полосы) за счет использования второй стороны диэлектрического материала, на которой может быть размещена аналогичная структура с параметрами, обеспечивающими ее работу на отстоящей частоте. Кроме того, возможно рассмотрение вариации технологии изготовления с использованием проводящих чернил вместо медного слоя и резисторов, что позволит использовать подобные структуры на значительно более высоких частотах [10].

\section{Финансирование работы}

Исследование выполнено при финансовой поддержке Российского фонда фундаментальных исследований, Правительства Красноярского края и Красноярского краевого фонда науки в рамках научного проекта № 2047-240003.

\section{Конфликт интересов}

Авторы заявляют, что у них нет конфликта интересов.

\section{Список литературы}

[1] Trimble Zephyr 3 (datasheet) (Trimble, Inc., 2017). https://geospatial.trimble.com/

[2] D.R. Smith, W.J. Padilla, D.C. Vier, S.C. Nemat-Nasser, S. Schultz, Phys. Rev. Lett., 84 (18), 4184 (2000). DOI: $10.1103 /$ PhysRevLett.84.4184

[3] R.A. Shelby, D.R. Smith, S. Schultz, S.C. Nemat-Nasser, Appl. Phys. Lett., 78 (4), 489 (2001). DOI: 10.1063/1.1343489

[4] J. Tak, Y. Lee, J. Choi, J. Electromagn. Eng. Sci., 13 (1), 1 (2013). DOI: 10.5515/JKIEES.2013.13.1.1.1

[5] A. Yelizarov, A. Kukharenko, in German Microwave Conf. (GeMiC) (Bochum, Germany, 2016), p. 271.

DOI: 10.1109/GEMIC.2016.7461608

[6] Frequency response of thin film chip resistors (Vishay Intertechnology, Inc., 2009), document number 601074, revision: 04-Feb-09. https://www.vishay.com/

[7] X. Chen, T.M. Grzegorczyk, B.-I. Wu, J. Pacheco, Jr., J.A. Kong, Phys. Rev. E, 70 (1), 016608 (2004). DOI: 10.1103/PhysRevE.70.016608

[8] J.M. Tranquilla, J.P. Carr, H.M. Al-Rizzo, IEEE Trans. Antennas Propagat., 42 (7), 905 (1994). DOI: $10.1109 / 8.299591$

[9] Springer handbook of global navigation satellite systems, ed. by P.J.G. Teunissen, O. Montenbruck (Springer, Berlin, 2016). DOI: 10.1007/978-3-319-42928-1_4

[10] P. Tiwari, S.K. Pathak, V.P. Anitha, in 2020 IEEE Int. Conf. on computational electromagnetics (ICCEM) (IEEE, 2020), p. 243. DOI: 10.1109/ICCEM47450.2020.9219409 